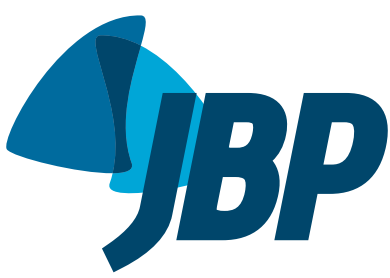

\section{The difficult task of searching for tools that help predict mechanical ventilator weaning success}

\author{
Bruno do Valle Pinheiro ${ }^{1}$
}

Mechanical ventilation (MV) is one of the most common supportive measures employed in ICU and is fundamental in maintaining life under certain conditions. ${ }^{(1)}$ However, MV can be associated with serious complications, such as pneumonia, tracheobronchitis, critical illness neuropathy and myopathy, delirium, barotrauma, and MV-induced lung injury, making its interruption desirable once the patient is able to breathe spontaneously in a safe manner and without need of a tracheal cannula. ${ }^{(2)}$ This process of disconnecting the patient from the ventilator is designated weaning.

Weaning remains one of the great challenges of MV, especially because it is impossible to predict, with the desired accuracy, whether extubation will be successful or whether reintubation will be necessary. The rates of patients who undergo unscheduled extubation and are successfully weaned range from $25 \%$ to $75 \%$; these data show that exaggerated conservatism may delay MV weaning in some cases. However, the mean rates of reintubation after elective extubation remain between $10 \%$ and $12 \%$, regardless of the indices used to in order to predict weaning success. ${ }^{(4)}$ Nevertheless, these are mean values and certainly vary depending on the complexity of MV weaning: it can be simple - patients extubated after a first spontaneous breathing trial (SBT) - difficult - patients who fail on the first SBT and require up to three SBTs or up to 7 days after the first weaning trial - or prolonged - patients requiring more than three SBTs or more than 7 days after the first weaning trial.

Among the causes of weaning failure, especially in difficult and prolonged cases, it is worth noting cardiac dysfunction, associated or not with fluid overload. When the patient spontaneously breathe without the help of positive pressure of the ventilatory support, either during a T-piece trial or after extubation, the negative intrathoracic pressure during inhalation promotes an increase in venous return, with a consequent increase in the preload of the right and left ventricles, as well as a decrease in the left ventricular ejection pressure gradient, causing an increase in left ventricular afterload. At the same time, right after spontaneous breathing is initiated, there may be an increase in adrenergic tone, with increased levels of catecholamines and increases in left ventricular preloads and afterloads. These changes altogether increase oxygen consumption by the myocardium and may even generate ischemia in patients with previous coronary disease. Another possible consequence is the inability of the heart to deal with the increases in preload and afterload, resulting in increased filling pressures and pulmonary congestion. Pulmonary congestion increases the work of breathing and may be responsible for MV weaning failure. ${ }^{(6)}$

Given the importance of cardiac dysfunction and hypervolemia in weaning failure, it is expected that the identification of these conditions can be useful in the evaluation of these patients. In this sense, Antonio et al., (7) in the current issue of the JBP, evaluated whether the presence of signs of pulmonary congestion on chest $X$-rays correlated with SBT failure. To that end, the authors evaluated patients older than 18 years of age undergoing MV for more than $24 \mathrm{~h}$, depending on their clinical or surgical conditions. The patients were evaluated daily and were considered eligible for weaning if the cause of their respiratory failure improved and if they had good level of consciousness, adequate gas exchange, absence of respiratory acidosis, hemodynamic stability, and a rapid shallow breathing rate $\leq 105$ breaths/min/L. In such cases, a T-tube was placed for 30-120 min, and the following signs of failure were observed: RR $>30$ breaths/ min; arterial oxyhemoglobin saturation < 90\%; use of accessory muscles; HR > 140 bpm; systolic blood pressure $<90 \mathrm{mmHg}$ or $<20 \%$ of basal levels; and altered level of consciousness. The presence of any of these findings indicated SBT failure, whereas the absence of all of the signs meant SBT success, and extubation was carried out. A radiologist, blinded to the SBT result, evaluated the chest X-ray performed within $24 \mathrm{~h}$ prior to the trial and used a radiological score, described by Shochat et al., ${ }^{(8)}$ in order to assess pulmonary congestion.

The authors evaluated 170 patients, the majority of whom had simple weaning - 78.3\% were extubated on first attempt, and the duration of MV before weaning was 4 days (interquartile range [IQR]: 2-4 days) among those who had SBT success and 6 days (IQR: 4-11 days) among those who had SBT failure. The radiological score was not able to discriminate SBT results; the scores were similar between the patients showing SBT success or SBT failure (median = 3 days; IQR: 2-4 days) in both groups. ROC curve analysis revealed no cut-off point that accurately discriminated between SBT success and SBT failure. The results led the authors to conclude correctly that there is no indication to perform chest X-rays in order to evaluate pulmonary congestion as an additional tool to recommend the use of SBT in patients who meet the commonly accepted criteria to start the trial.

In some respects, this negative result could already be expected. The vast majority of patients had simple weaning; therefore, failure rates were low. Failure can occur due to various causes, cardiovascular failure being only one of them. In addition, among the patients studied in that cohort, less than half had systolic or diastolic 
dysfunction, which would be risk factors for weaning failure due to cardiovascular disease or hypervolemia. However, this negative result does not rule out the possibility that the evaluation of cardiac dysfunction or hypervolemia may be useful as a predictor of weaning success. In this sense, at least two points deserve to be discussed. The first point is whether such an assessment is necessary before SBT is performed in each and every patient. Performing additional evaluations in patients with a low probability of failure can be only a delaying factor in extubation, increasing the chances of MV complications. Therefore, it would be interesting to study a population at a greater risk of failure, even with an increased risk of cardiovascular failure or hypervolemia. Some criteria that could define this population would be the classification of weaning as difficult or prolonged, history of heart disease, or other risk factors, such as advanced age. The second point is whether a chest X-ray is the ideal tool for this sort of investigation or whether other options have higher yields. Among these options, some studies have demonstrated the usefulness of echocardiography and B-type natriuretic peptide quantification in identifying patients who fail SBT due to heart disease. ${ }^{(9,10)}$

In summary, another study showed the ineffectiveness of an isolated parameter in predicting SBT success or SBT failure; in this case, a radiological score for pulmonary congestion. Although the parameter itself might be inadequate, it should be considered that the result might have been due to the population studied, which consisted of patients who had simple weaning, with a low probability of weaning failure. Increasing the number of predictors is not only unnecessary but can lead to delayed extubation, as the authors have properly discussed.

\section{REFERENCES}

1. Esteban A, Ferguson ND, Meade MO, Frutos-Vivar F, Apezteguia C, Brochard L, et al. Evolution of mechanical ventilation in response to clinical research. Am J Respir Crit Care Med. 2008;177(2):170-7. https://doi.org/10.1164/rccm.200706-8930C

2. Boles JM, Bion J, Connors A, Herridge M, Marsh B, Melot C, et al Weaning from mechanical ventilation. Eur Respir J. 2007;29(5):1033 56. https://doi.org/10.1183/09031936.00010206

3. Kiekkas P, Aretha D, Panteli E, Baltopoulos GI, Filos KS Unplanned extubation in critically ill adults: clinical review. Nursing Crit Care. 2012;18(3):123-34. https://doi.org/10.1111/j.14785153.2012.00542.x

4. Peñuelas $\mathrm{O}$, Thille AW, Esteban A. Discontinuation of ventilatory support: new solutions to old dilemmas. Curr Opin Crit Care. 2015;21(1):74-81. https://doi.org/10.1097/MCC.0000000000000169

5. Perren A, Brochard L. Managing the apparent and hidden difficulties of weaning from mechanical ventilation. Intensive Care Med. 2013;39(11):1885-95. https://doi.org/10.1007/s00134-013-3014-9

6. Teboul JL. Weaning-induced cardiac dysfunction: where are we today? Intensive Care Med. 2014;40(8):1069-79. https://doi org/10.1007/s00134-014-3334-4

7. Antonio ACP, Teixeira C, Castro PS, Zanardo AP, Gazzana MB, Knorst M. Radiological signs of pulmonary congestion do not predict failed spontaneous breathing trial. J Bras Pneumol. 2017;43(4):253-58.

8. Shochat M, Shotan A, Trachtengerts V, Blondheim DS, Kazatsker M, Gurovich V, et al. A novel radiological score to assess lung fluid content during evolving acute heart failure in the course of acute myocardial infarction. Acute Card Care. 2011;13(2):81-6. https://doi org/10.3109/17482941.2011.567279

9. Lamia B, Maizel J, Ochagavia A, Chemla D, Osman D, Richard C, et al. Echocardiographic diagnosis of pulmonary artery occlusion pressure elevation during weaning from mechanical ventilation. Crit Care Med. 2009;37(5):1696-701. https://doi.org/10.1097/ CCM.0b013e31819f13d0

10. Mekontso Dessap A, Roche-Campo F, Kouatchet A, Tomicic V Beduneau G, Sonneville $R$, et al. Natriuretic peptide-driven fluid management during ventilator weaning: a randomized controlled trial. Am J Respir Crit Care Med. 2012;186(12):1256-63. https://doi. org/10.1164/rccm.201205-09390C 